\title{
Bardeen-Petterson Effect and Broad HI Profiles of Seyferts
}

\section{Rumen Bachev}

Institute of Astronomy, BAS, 72 Tsar. Shose, 1784 Sofia, Bulgaria. Email: bachevr@astro.bas.bg

A thin accretion disk could be not only the energy source of AGN but also the matter producing broad emission lines of Seyfert 1 type nuclei (Dumont \& Collin-Souffrin, $1990 \mathrm{~B}$ ). A possible mechanism for this is reprocessing of central hard X-ray radiation by the outer (at $10^{2-5} \mathrm{RG}, \mathrm{RG}$ is the Schwarzschild radius), low-temperature regions of the disk. This mechanism is effective enough especially if the disk is a non-planar structure (a warped or twisted disk), when the outer parts could be directly seen from the centre. An accretion disk around a Kerr black hole could be twisted if the angular momentum of the accreting gas is initially not aligned with the rotation axis of the hole. Due to the differential Lense-Thirring precession of orbits around a Kerr black hole, a viscous disk is a steady but non-planar structure. This is the well-known Bardeen-Petterson effect (Bardeen \& Petterson, 1975). Near the hole, at distances $R<R B P$, where RBP is the Bardeen-Petterson radius, the flow is aligned with the equatorial plane of the black hole, while at larger distances it is tilted to its initial orbital plane (Fig. 1).

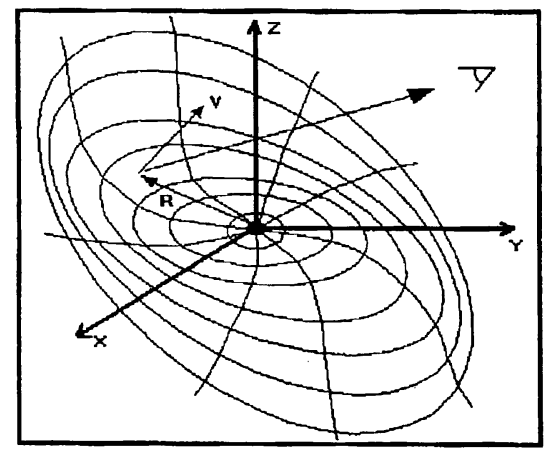

Figure 1. The illumination of a non-planar disk. Each gas volume reemits a part of the ionising continuum if direct illumination is possible. To a distant observer, the intensity and frequency shift of this emission depend on the velocity $(\mathbf{V})$, the orientation and intercepted amount of hard radiation as well as on the reprocessing properties of the volume. The twisting structure of the disk is described by two Eulerian angles - slowly varying functions of $\boldsymbol{R}$ and the initial inclination angle. The solution, describing disk twisting, derived by Hatchett et al. (1981) is used here. 
If it exists, an accretion disk in AGN should be non-planar in most cases as there is no reason to suppose that the angular momentum of accreting matter and the black hole spin axis are always coplanar. We studied numerically the profiles of the broad low-ionisation lines $\left(\mathrm{H}_{\beta}\right)$ produced by an irradiated twisted disk.

Reprocessing properties of the disk layers are taken into account following closely Collin-Souffrin and Dumont (1990). The central hard X-ray source is proposed to be point-like, at zero height above the disk plane. Since the twisted disk is anisotropically irradiated and just a part of the disk emits towards the observer's direction (Fig. 2), the profiles of the broad lines are generally nonsymmetric and frequency-shifted with respect to the systemic velocity (Fig. 3). If the disk is transparent (a case not considered here), emission from the lower disk surface may come and profiles would be double-peaked and almost symmetric.

Because such frequency displaced, asymmetric profiles are seldom observed (several objects with similar profiles are known, for instance - 3C 227 and Mkn 668 , Eracleous \& Halpern, 1994), one may conclude that the bulk of the line emission of AGN arises from some more or less spherical or conical structure (a system of clouds or star atmospheres, jet, etc.) but not from a disk. In this case, the presence of an illuminated twisted accretion disk will cause only line asymmetry or a frequency displaced secondary peak. The absence of such asymmetry or displaced peaks may probably be an indication for the presence of non-rotating black holes in the centres of active galaxies when the disks are planar and the disk emission symmetric and double-peaked (Dumont \& CollinSouffrin, 1990 B). Asymmetrical double-peaked profiles could be observed from a warped disk if the ionising source is located far above the disk plane or a hot diffuse medium reflecting hard radiation back towards the disk is present (models not considered here). In this case the central parts of the disk will be illuminated isotropically and will produce the main double-peaked profile. 


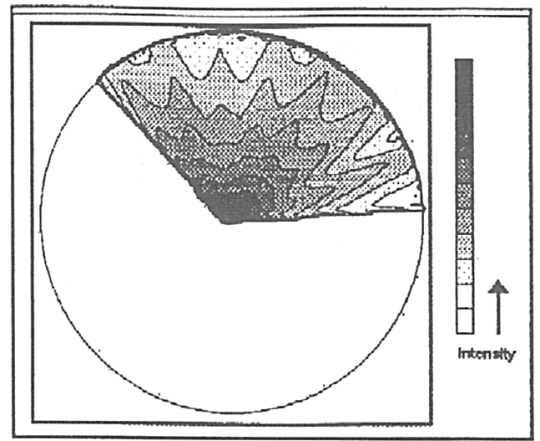

Figure 2. A schematic map of the $\mathrm{H}_{\beta}$ radiation flux from a selfirradiated warped disk. Standard thin Shakura-Sunyaev $\alpha$-disk model is accepted. The initial inclination angle of the flow is $10^{\circ}$. Relative units are used to represent the emission-line flux from the disk. Black hole mass is $10^{8} \mathrm{M}_{\odot}$, accretion rate $0.1 \mathrm{M}_{\odot} \mathrm{yr}^{-1}$. Dimensionless spin parameter $a=1$, viscosity parameter $\alpha=0.1$. Bardeen-Petterson radius (the radial distance where the infall time scale is comparable to the precession time scale) is about $300 \boldsymbol{R}_{\mathrm{G}}$. Only the inner part $(\boldsymbol{R}<100$ $\boldsymbol{R}_{\mathrm{BP}}$ ) of the disk is presented here.

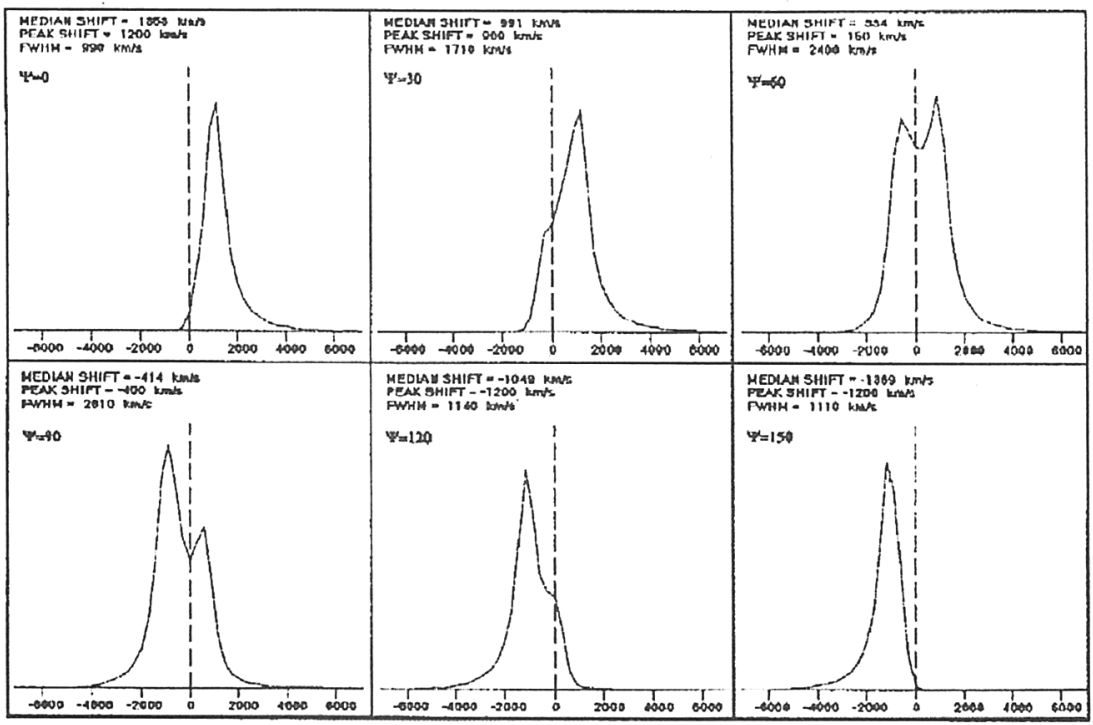

Figure 3. Line profiles of $\mathrm{H}_{\beta}$ emission from the disk on Fig. 2 and different orientation angles. The observer is tilted at an angle $30^{\circ}$ with respect to the black hole equatorial plane. The azimuthal angle of the observer $(\Psi)$ along the disk plane is respectively $0,30, \ldots, 150^{\circ}$. The disk is extended up to $100 \boldsymbol{R}_{\mathrm{BP}}$. 


\section{References}

Bardeen J. M., Petterson J. A., 1975, ApJ, [195], L65

Collin-Souffrin S., Dumont A. M., 1990, A\&A, [229], 292

Dumont A. M., Collin-Souffrin S., 1990, A\&A, [229], 302, A

Dumont A. M., Collin-Souffrin S., 1990, A\&A, [229], 313, B

Eracleous M, Halpern J. P.,1994, ApJS, [90], 1

Hatchett S. P., Begelman M.C. \& Sarazin C.L., 1981, ApJ. [247], 677 